\title{
Radio continuum monitoring of the extreme carbon star IRC+10216
}

\author{
K. M. Menten ${ }^{1}$, M. J. Reid ${ }^{2}$, E. Krügel ${ }^{1}$, M. J. Claussen ${ }^{3}$, and R. Sahai ${ }^{4}$ \\ 1 Max-Planck-Institut für Radioastronomie, Auf dem Hügel 69, 53121 Bonn, Germany \\ e-mail: kmenten,p309ekr@mpi fr-bonn.mpg.de \\ 2 Harvard-Smithsonian Center for Astrophysics, 60 Garden Street, Cambridge, MA 02138, USA \\ e-mail: reid@cfa.harvard.edu \\ 3 National Radio Astronomy Obsrvatory, Array Operations Center, PO Box O, Socorro, NM 87801, USA \\ e-mail: mclausse@nrao.edu \\ 4 Jet Propulsion Laboratory, MS 183-900, 4800 Oak Grove Drive, Pasadena, CA 91109, USA \\ e-mail: raghvendra.sahai@jpl.nasa.gov
}

Received 2 November 2005 / Accepted 15 March 2006

\begin{abstract}
We describe Very Large Array observations of the extreme carbon star IRC+10216 at 8.4, 14.9, and $22.5 \mathrm{GHz}$ made over a two year period. We find possible variability correlated with the infrared phase and a $\mathrm{cm}$ - to sub-millimeter wavelength spectral index very close to 2. The variability, observed flux densities, and upper limit on the size are consistent with the emission arising from the stellar photosphere or a slightly larger radio photosphere.
\end{abstract}

Key words. stars: carbon - stars: AGB and post-AGB - radio continuum: stars - techniques: interferometic

\section{Introduction}

The "extreme" carbon star IRC+10216 (CW Leonis) was discovered in the early days of modern infrared astronomy (Becklin et al. 1969). The star is one of the brightest near- and mid-infrared sources (LeBertre 1987 and references therein). IRC +10216 has an extremely rich molecular spectrum arising from a dense envelope created by a powerful mass outflow (Cernicharo et al. 2000). These attributes, together with its proximity (distance of $\approx 130 \mathrm{pc}$ ) and its prodigious mass-loss rate (1.5-3.3 $\left.\times 10^{-5} M_{\odot} \mathrm{yr}^{-1}\right)$, make IRC +10216 a "keystone" object for which many aspects of (carbon-star) asymptotic giant branch (AGB) evolution can be studied in detail. IRC+10216's properties are summarized in Table 1.

IRC +10216 has been detected at wavelengths of 1.5 and $2 \mathrm{~cm}$ (Sahai et al. 1989; Drake et al. 1991). From Very Large Array (VLA) observations it should be possible to measure its size, position (which is extremely difficult at other wavelengths owing to absorption and scattering), proper motion, and the spectral index of its radio emission. Very Large Array data can strongly constrain our information on the star's nature.

This paper has the following structure: in Sect. 2, we describe six-epoch, three-wavelength VLA observations. These, and an additional dataset (published by Drake et al. 1991), were retrieved from the VLA archive. The properties of the continuum emission are discussed in Sect. 3. In particular, we address the variability, spectral index, absolute position, and source morphology. In Sect. 4 we synthesize these results to reach conclusions on the nature of the radio emission.

\section{VLA observations and data reduction}

\subsection{1-1993 observations}

The data reported here were taken by two of us (RS \& MC) with the NRAO Very Large Array (VLA) ${ }^{1}$. We analyzed observations made between 1991 June 1 and 1993 June 9, spaced typically by 3 to 4 months. Observations were made at $8.4,14.9$, and $22.5 \mathrm{GHz}$ (X-, U-, and K-band, respectively) ${ }^{2}$. Details are given in Table 2. A typical observing run consisted two 20-28 min long X-and U-band observations, preceded and followed by observations of the calibrator $0953+254$. Because of the generally short coherence times at the highest frequency, the K-band scans were shorter ( $\approx 15$ min duration) and more scans were made in this band. At the end of each run, we observed the absolute flux density calibrator 3C 286.

The flux density of $0953+254$ was determined by comparing its visibility amplitude with that of 3C 286. The elevations of 3C 286 were comparable to that of $0953+254$, minimizing systematic amplitude calibration errors due to elevationdependent antenna gain. By comparing the variations of the flux densities for several scans of $0953+254$, we estimate our flux density scale is accurate to within $10 \%$ at X-band, $15 \%$ at U-band, and $20 \%$ at K-band. Data from one epoch (1992 October 2) suffered serious instrumental difficulties and were discarded. Table 2 contains the measured flux densities of $0953+254$. Complex gain correction factors were interpolated

1 The National Radio Astronomy Observatory (NRAO) is operated by Associated Universities Inc., under a collaborative agreement with the US National Science Foundation.

2 To avoid confusion, throughout the paper radio spectral bands are referred to in roman font and infrared bands in italics. 
Table 1. IRC+10216 - fundamental parameters.

\begin{tabular}{lll}
\hline \hline Name & IRC+10216 & Reference \\
\hline Aliases & CW Leonis, RAFGL 1381 & SIMBAD $^{a}$ \\
& IRAS 09452+1330 & \\
$\alpha_{\text {J2000 }}$ & $09^{\mathrm{h}} 47^{\mathrm{m}} 57^{\mathrm{s}} .392^{b}$ & \\
$\delta_{\mathrm{J} 2000}$ & $+13^{\circ} 16^{\prime} 43^{\prime \prime} 63^{b}$ & \\
Spectral type & $\mathrm{C}$ & \\
Effective temperature, $T_{\text {eff }}$ & $2000 \mathrm{~K}^{c}$ & Groenewegen (1997) \\
Luminosity, $L_{\star}$ & $8.2 \times 10^{5}[D(\mathrm{kpc})]^{2} L_{\odot}{ }^{d}$ & Groenewegen (1997) \\
Mass loss rate, $M_{\odot}$ & $1.5-3.3 \times 10^{-5} M_{\odot} \mathrm{yr}^{-1} e$ & $\begin{array}{l}\text { Crosas \& Menten (1997), } \\
\text { Groenewegen et al. (1998) }\end{array}$ \\
Distance, $D^{d}$ & & Crosas \& Menten (1997), \\
& $110-150 \mathrm{pc}$ & Groenewegen et al. (1998) \\
Expansion velocity & $16 \mathrm{~km} \mathrm{~s}^{-1}$ & Loup et al. (1993) \\
LSR velocity, $v_{\mathrm{LSR}}$ & $-22 \pm 1.5 \mathrm{~km} \mathrm{~s}^{-1}$ & Loup et al. (1993) \\
Heliocentric velocity, $v_{\text {hel }}$ & $-15 \mathrm{~km} \mathrm{~s}^{-1}$ & \\
\hline
\end{tabular}

${ }^{a}$ http://simbad.u-strasbg.fr/. ${ }^{b}$ Position derived from 1993 January 26 VLA data. The estimated uncertainty is 25 milli arcseconds (see Sect. 3.3). ${ }^{c}$ Groenewegen (1997) calculates a range of models resulting from a range of values for this parameter; we adopt $2000 \mathrm{~K}$, which is used in most of his models. ${ }^{d}$ Assuming $T_{\text {eff }}=2000 \mathrm{~K} .{ }^{e}$ The listed mass loss rate and distance estimates represent the range of values derived from modeling of multi-line $\mathrm{CO}$ observations described in the referenced studies.

Table 2. Log of IRC+10216 multiband VLA observations.

\begin{tabular}{lllllllll}
\hline \hline JD 2440000+ & Civil Date & Array & \multicolumn{2}{c}{$S(\mathrm{~B} 0953+254)(J y)$} & \multicolumn{3}{c}{$S(\mathrm{IRC}+10216)(\mathrm{mJy})$} \\
& & & X-band & U-band & K-band & X-band & U-band & K-band \\
\hline 8410 & 1991 June 01 & $\mathrm{D}(\rightarrow \mathrm{A})$ & $1.80(0.01)$ & $1.90(0.05)$ & $2.27(0.07)$ & $0.92(0.07)$ & $2.67(0.39)$ & $7.10(0.68)$ \\
8524 & 1991 September 24 & $\mathrm{A} \rightarrow \mathrm{B}$ & $1.80(0.08)$ & $2.02(0.13)$ & $2.40(0.13)$ & $0.48(0.07)$ & $2.11(0.55)$ & $3.87(1.20)$ \\
8652 & 1992 February 09 & $\mathrm{CnB}$ & $1.56(0.01)$ & $1.58(0.01)$ & $1.51(0.01)$ & $0.82(0.04)$ & $2.23(0.17)$ & $4.24(0.33)$ \\
8772 & 1992 May 30 & $\mathrm{C} \rightarrow \mathrm{D}$ & $1.54(0.01)$ & $1.52(0.02)$ & $1.33(0.03)$ & $0.86(0.07)$ & $2.42(0.20)$ & $3.18(0.67)$ \\
9014 & 1993 January 26 & $\mathrm{A}$ & $1.58(0.03)$ & $1.55(0.04)$ & $1.60(0.05)$ & $0.60(0.04)$ & $2.00(0.31)$ & $5.33(1.03)$ \\
9143 & 1993 June 09 & $\mathrm{CnB}$ & $1.72(0.01)$ & $1.69(0.05)$ & $1.77(0.24)$ & $0.48(0.04)$ & $1.77(0.21)$ & $3.86(0.60)$ \\
\hline Average & & - & - & - & - & $0.67(0.03)$ & $2.14(0.18)$ & $4.20(0.46)$ \\
\hline
\end{tabular}

The first six lines present the results of our VLA measurements epoch by epoch. The first and second columns give the Julian and civil dates, respectively. The third column gives the array configuration. Two letters connected by an arrow denote epochs when the array was in transition from one to another configuration; $\mathrm{CnB}$ denotes a mixed $\mathrm{C}$ - and $\mathrm{D}$-configuration. The forth to sixth columns give the bootstrapped flux density of the phase calibrator B0953+25 at X-, U-, and K-band respectively, while the seventh to ninth columns give the same for IRC+10216. The quoted uncertainties are formal errors from JMFIT. We estimate additional systematic errors of 10, 15, and $20 \%$ for X-, U-, and K-band data, respectively. Based on the formulae given by Baars et al. (1977), the absolute flux calibrator 3C 286 was assumed to have flux densities of 5.21, 3.46, and $2.52 \mathrm{Jy}$ at X-, U-, and K-band. For each band, data from two $50 \mathrm{MHz}$ wide channels were taken in right- and left-hand circular polarization. The center frequencies were $8.439,14.940$, and $22.460 \mathrm{GHz}$ for the X-, U-, and K-band data. For the first epoch (1991 June 1, JD 2448410) only visibilities within a $u, v$-range of $50 \mathrm{k} \lambda$, corresponding to D-array antenna separations, were considered. Data from one more epoch (1992 October 2, JD 2448897) had to be discarded due to instrumental problems. The bottom line gives weighted averages, with individualepoch uncertainties estimated by adding quadratically the formal uncertainties from JMFIT with estimated absolute calibration uncertainties.

from the amplitude and phase of $0953+254$ to the times of the IRC+10216 data.

We made maps using the AIPS task IMAGR and fit elliptical Gaussian models to the images. The integrated flux densities are listed in Table 2. Because IRC +10216 is weak, self calibration was not possible, and the "fast-switching" technique, routinely employed now, had not been developed at the time of our observations. Nevertheless, during one epoch (1993 January 26) when the array was in the A-configuration, superb phase coherence persisted throughout the observations. We use those data to establish the source position (in Sect. 3.3).

\subsection{Other VLA data}

VLA A-configuration observations of IRC+10216 have been reported by Drake et al. (1991). We re-analyzed their 1987 June 2 and 3 U-band data to compare with our U-band data. Fitting an elliptical Gaussian using the AIPS task JMFIT, we find the source to be nearly unresolved, with upper limits on the deconvolved size of 95 milliarcesconds (mas) and a peak brightness and integrated flux density of $1.29 \pm 0.15 \mathrm{mJy} \mathrm{beam}^{-1}$ and $1.40 \pm 0.27 \mathrm{mJy}$, respectively. The latter value can be compared with our observed range of integrated U-band flux densities of 1.77 to $2.67 \mathrm{mJy}$ (see Table 2). Since we used identical calibration procedures for both datasets and the same $a b$ solute flux calibrator, this discrepancy is probably real. Since the 1987 data employed a secondary gain calibrator nearer to IRC +10216 than used for our data, one would expect that the 1987 data should yield a greater flux density than the 1993 data, if random atmospheric phase fluctuations degrade the imaging.

In order to estimate of the absolute position accuracy, we made images from the the June 2 and 3 data separately. The positions of the fitted Gaussians differed by 18 mas in right ascension and 10 mas in declination, testifying to the excellent phase coherence during these observations. Assuming that large-scale atmospheric effects, which usually limit astrometric accuracy, were independent for these two days, this indicates an uncertainty of $\approx 20$ mas in each coordinate. 


\section{Properties of the radio emission}

\subsection{Variability}

In Fig. 1 we show the flux densities of IRC+10216 listed in Table 2. The error bars include contributions from random noise and systematic calibration uncertainties. The data show probable variability. We fit the flux densities of IRC +10216 with a model that includes a constant and a sinusoidally varying component. Rather than fitting the data from each band separately, we assume that a constant spectral index, $\alpha$, describes the difference in flux density with observing frequency. Specifically, the model for the flux density, $S(v, t)$, at a frequency, $v$, and time, $t$, is given by the following:

$S(v, t)=\left(S\left(v_{0}\right)+\Delta S\left(v_{0}\right) \cos (\Delta t / P)\right)\left(v / v_{0}\right)^{\alpha}$,

where $S\left(v_{0}\right)$ and $\Delta S\left(v_{0}\right)$ are the constant and amplitude of the time varying flux density at a reference frequency $v_{0}$ (set to $10 \mathrm{GHz}), \Delta t$ is the time offset from maximum $\left(t_{\max }\right)$, and $P$ is the period.

A weighted least-squares fit to the data yielded the following results: $S\left(v_{0}\right)=1.02 \pm 0.14 \mathrm{mJy}, \Delta S\left(v_{0}\right)=0.25 \pm 0.09 \mathrm{mJy}$, $P=535 \pm 51 \mathrm{~d}, t_{\max }=(\mathrm{JD} 2440000+) 8841 \pm 24 \mathrm{~d}$, and $\alpha=1.88 \pm 0.14$. This fit has a reduced $\chi^{2}$ per degree of freedom of 1.6, and the parameter uncertainties are the formal $1 \sigma$ values scaled by $\sqrt{1.6}$. Thus, IRC+10216 appears to display flux density variations of $\pm 25 \%$ at a significance level of nearly $3 \sigma$. The variability will be further discussed in the Appendix.

IRC+10216 shows strong periodic variability at infrared wavelengths (Dyck et al. 1991; Le Bertre 1992). Dyck et al. determine a period of $638 \mathrm{~d}$ at (infrared) $K$-band, while Le Bertre's measurements in the $J-, H-, K-, L^{\prime}$-, and $M$-bands yield values between 636 and $670 \mathrm{~d}$. If we assume the infrared period has a $5 \%$ uncertainty, then the cm-wave period of $535 \pm 51 \mathrm{~d}$ differs from the infrared period by about $2 \sigma$. The $\mathrm{cm}$-wave maximum occurs at $8841 \pm 24$, whereas the infrared maximum (extrapolated by about $1200 \mathrm{~d}$ ) would be predicted to occur near 8770 with an estimated uncertainty of about \pm 50 . Thus, the $\mathrm{cm}$-wave minus infrared maxima differ by $71 \pm 71 \mathrm{~d}$ and are consistent within their joint uncertainty.

\subsection{Spectral index}

As discussed above, the radio emission from IRC+10216 is probably variable. Therefore, we calculate the X-U-K-band spectral index (SI) for each epoch and find values between 1.6 and 2.2, with uncertainties of up to $20 \%$. These daily SIs are consistent with a constant value, and a variance-weighted average of these indices is $1.92 \pm 0.11$. We also listed in Table 2 variance-weighted, time-averaged flux densities for each band. Using these to determine a SI yields $1.91 \pm 0.11$. The close correspondence between the daily and time averaged SIs suggests that the spectral index is not strongly variable.

The $\mathrm{cm}$-wave spectral index obtained by least-squares fitting of all of the data simultaneously with the model described above is $1.88 \pm 0.14$. This value is consistent with those observed for Mira variables and with the value of 1.86 for a model of Miras Reid \& Menten (1997a). However, the cm-wave spectral index is also consistent with a value of 2.0 for optically-thick black-body emission.

At millimeter- and sub-millimeter wavelengths, flux densities measured with single-dish telescopes (with $>10^{\prime \prime}$ FWHM beams) contain contributions from the star as well as from the extended dust envelope. If the measurements are made with
Julian Date $(2440000+)$
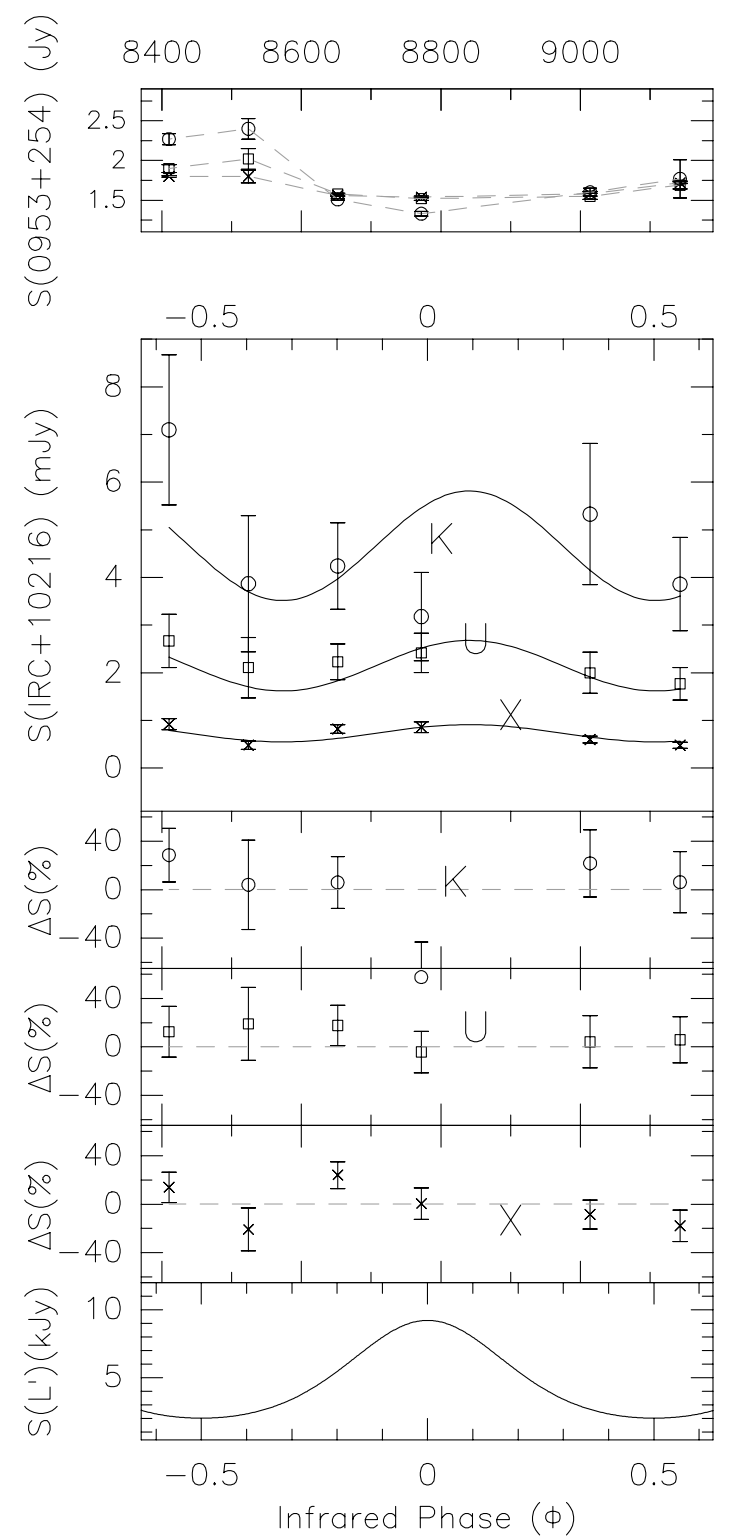

Fig. 1. Radio flux densities of IRC+10216 from 1991 June 1 to 1993 June 8 (second panel from top) compared to that of the calibrator $0953+254$ (top panel). For both sources the X-, U-, and K-band integrated flux densities from Gaussian fits are plotted bottom to top. Note the different flux density scales. Error bars represent the quadratic sum of formal fitting errors and estimates of the errors due to absolute flux density calibration $(10,15$, and $20 \%$ for the X-, U-, and K-band data points). In the IRC+10216 panel, the solid lines represent the results of the model calculations described in the text. The third to fifth panels from top show K-, U- and X-band residuals in percent-for each epoch the flux density predicted by the model was subtracted from the measured value and divided by the model value. X-, U-, and K-band data points are represented as crosses, squares, and circles, respectively. In all panels the same time range is shown. The abscissa gives Julian day number in the top panel and infrared ( $L^{\prime}$-band) stellar phase, $\Phi$, in the others. The date of maximum infrared light $(\Phi=0)$ was calculated from the value determined by LeBertre 1992 (JD 2447483), using his period of $649 \mathrm{~d}$. The lowermost panel represents the $L^{\prime}$-band $(3.76 \mu \mathrm{m})$ light curve converted to kJy units (see Fig. 2k of Le Bertre 1992).

wideband bolometer detectors, circumstellar molecular emission may also contribute $\approx 30 \%$ to the total flux density (Walmsley et al. 1991; Groenewegen 1997). Whereas emission by dust 


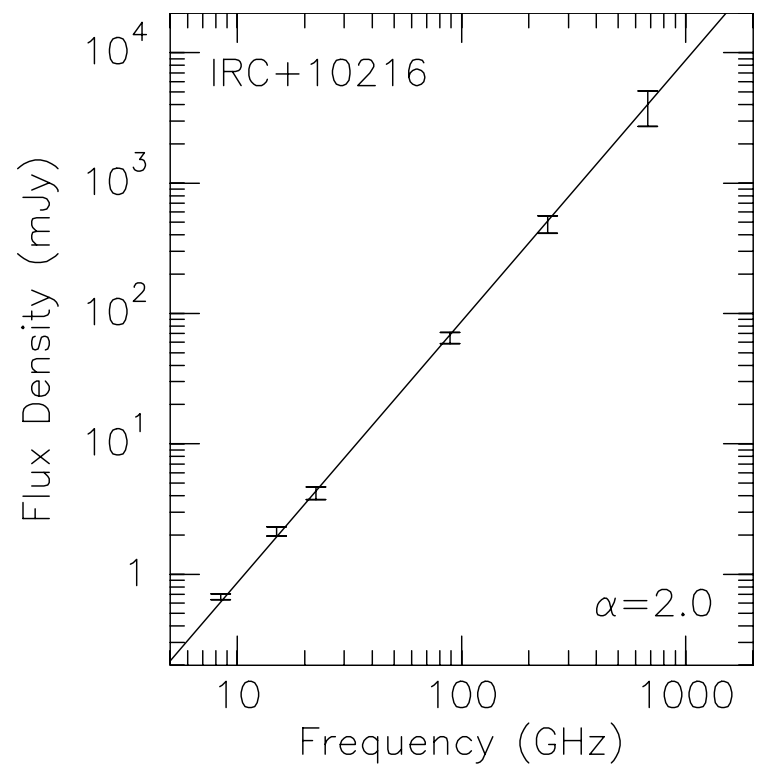

Fig. 2. Interferometric flux densities of IRC+10216 from $\mathrm{cm}$ to sub$\mathrm{mm}$ wavelengths. The $\mathrm{X}-, \mathrm{U}-$, and $\mathrm{K}$-band $\mathrm{cm}$-wave data points are the weighted averages of data from six epochs (see Table 1). These error bars are quadratic sums of formal uncertainties and absolute calibration errors, which are assumed to be 10,15 , and $20 \%$ for the X-, U-, and K-band data, respectively. The error bars of the $\mathrm{mm}$ and submm wavelength data are discussed in the text. The straight line has a slope of 2.0, the blackbody value. A least squares fit to all the shown data delivers a slope of $1.96 \pm 0.04$.

dominates in the submillimeter region on large scales (see Fig. 4), it is negligible at cm-wavelengths. Based on the model discussed in the Appendix, we expect that the dust envelope contributes only $\sim 0.1 \mathrm{mJy}$ to the total flux density of $\sim 2 \mathrm{mJy}$ in our VLA K-band measurements.

A few interferometric observations exist that should deliver accurate measurements of compact emission free of dust contamination. Also by selecting portions of the band largely free of molecular lines, nearly pure continuum emission can be measured. Lucas \& Guélin (1999), using the IRAM Plateau de Bure Interferometer (PdBI), find a "point source" at 89 and $242 \mathrm{GHz}$ with flux densities of $65 \pm 7$ and $487 \pm 70 \mathrm{mJy}$, respectively. (Note these uncertainties are much larger than the formal errors given in the cited paper; R. Lucas personal communication.) These authors also detect "extended" emission with flux densities of 12 and $57 \%$ of the point source values at 89 and $242 \mathrm{GHz}$, respectively, which they ascribe to dust emission from the inner envelope. Young et al. (2004) used the Smithsonian Sub-Millimeter Array (Ho et al. 2004) to image the $680 \mathrm{GHz}$ continuum emission of IRC +10216 . They also find a "compact, unresolved component" in their $\approx 2^{\prime \prime} F W H M$ beam with a flux density of $3.9 \pm 1.2 \mathrm{Jy}$. The flux densities discussed above are plotted in Fig. 2. A least-squares fit to all the data (cm through submillimeter) delivers an SI of $1.96 \pm 0.04$.

\subsection{Absolute position of the radio emission distribution}

During our 1993 January 26 observations, the VLA was in its most extended configuration and the phase coherence was excellent. Data from that epoch can be used to obtain meaningful images of the emission distribution and to make an astrometric position determination. To check the validity of the size information and the position accuracy, we determined, for the
K-band data, phase and amplitude calibration of only the first scan of $0953+254$ and then applied extrapolations of these solutions to subsequent scans of that source, which were spaced by $\approx 16 \mathrm{~min}$. As expected, the phase coherence degraded with elapsed time between the first and subsequent scans. However, in maps made from the $u v$-data of the second scan, taken 16 min after the first, the source showed $<3 \%$ amplitude degradation, a (formal) source size $<31$ mas and an offset of $<5$ mas in both coordinates from the nominal position. Since IRC+10216 scans were placed between $0953+254$ scans, we conclude that some, but probably little, blurring occurs due to deficient phase calibration. The test described above does not account for phase calibration errors caused by the fact that $0953+254$ is $>12 \mathrm{deg}$ away from IRC+10216. To take that into account, we increase our value for the systematic position uncertainty to 20 mas.

The position uncertainty may be dominated by statistical errors due to the modest signal to noise ratio of our continuum images. JMFIT delivered positions for the X-, U-, and K-band images differing by maximally 20 mas from each other, with formal errors of between 5 and 16 mas. The total uncertainty, obtained by quadratically adding the systematic and statistical uncertainty is 15 mas in each coordinate. The variance-weighted mean position and its uncertainty is given in Table 1. The position derived from the data of Drake et al. $(1991)$ is $(\alpha, \delta)_{\mathrm{J} 2000}=09^{\mathrm{h}} 47^{\mathrm{m}} 57^{\mathrm{s}} 382,+13^{\circ} 16^{\prime} 43^{\prime \prime}$. 61 , for which we estimate an uncertainty of 20 mas in each coordinate (see Sect. 2.2). The latter position (measured on 1987 June 2.5) is offset from the 1993 January 26 position by $(-146,-20)$ mas in the (East, North) directions. Taking these offsets at face value, we derive proper motion components of IRC+10216 of $(+26 \pm 6,+4 \pm 6)$ mas $\mathrm{yr}^{-1}$, respectively. A motion of that magnitude is plausible. For example, Mira (o Ceti), which is at a similar distance as IRC +10216 , has a larger proper motion of $(+33,-239)$ mas $\mathrm{yr}^{-1}$ measured by Hipparcos (Perryman et al. 1997).

The proper motion derived above is consistent with the upper limit of 30 mas $\mathrm{yr}^{-1}$ that Becklin et al. (1969) derive from a comparison of a Palomar Sky Survey plate taken in 1954 with a plate they took in 1969.

\subsection{Morphology}

Our best quality radio data (1993 Jan. 26) suggest an elongated flux distribution (position angle, PA, $82 \pm 5$ degrees; i.e. roughly east-west direction), with major and minor axes of $213 \pm 20$ and $73 \pm 20$ mas, respectively, which is significantly larger than the 1987 upper limits of 95 mas discussed in Sect. 2.2.

While an elongated source would be tantalizing, we have greater confidence in the 1987 than the 1993 data. The separation between IRC +10216 and $0952+179$, the phase calibrator used by Drake et al., is only 1.7 in right ascension and 4.4 in declination. In contrast, the phase calibrator used in 1993 is displaced by 2.1 in right ascension and a much larger distance, -12.2 , in declination from IRC +10216 . While it is difficult to tell conclusively, we believe that the source size observed in 1993 January may be affected by uncorrected residual phase errors stemming from the large angular separation of IRC +10216 and the calibrator.

Groenewegen (1996) takes the elongation found by Drake et al. (1991) at face value and argues for asphericity. We note that on scales of a few stellar radii, IRC+10216's morphology is highly asymmetric and changes with time. Multi-epoch $2.2 \mu \mathrm{m}$ speckle interferometry between 1995 and 1998 shows an asymmetric clumpy shell with two dominant components, A and B, 
whose separation grows from 191 to 265 milliarcseconds (mas) between 1995 and 1998 (Osterbart et al. 2000). $2.2 \mu \mathrm{m}$ Keck I aperture masking interferometry between 1997 and 1999 confirm the observed morphology (Tuthill et al. 2000). While both studies find motions in the material, these are inconsistent with simple spherical expansion and a bipolar flow. Osterbart et al. (2000) suggest that the star itself is identical to, or located near, component B. On the other hand, (Richichi et al. 2003), modeling their infrared lunar occultation data, favor an identification of the star with component A.

Future VLA observations outlined in Sect. 5, together with absolute infrared astrometry, may unambiguously establish the star's position relative to the clumpy components of its envelope. Also, future VLA array observations at 1.3 and $0.7 \mathrm{~cm}$ can definitively settle the question of IRC+10216's radio source size and morphology.

\section{The nature of the radio emission}

Groenewegen (1997) performed model calculations of IRC+10216's infrared through radio continuum emission. His models and assumptions under-predict our measured radio flux densities by a factor of $\approx 1.8$. A nearer distance of $\approx 100 \mathrm{pc}$ (instead of the $135 \mathrm{pc}$ assumed) would provide a better fit to the data. For X-and U-band, his models predict the emission to arise solely from the stellar photosphere, and at K-band the dust contribution from the whole envelope would add about $25 \%$ to the photospheric emission. Assuming an effective temperature of $2000 \mathrm{~K}$, a photospheric diameter of 70.2 mas is derived, which is consistent with our upper limit. As shown in the Appendix, assuming these values for temperature and diameter, IRC+10216's spectral energy distribution can be modeled successfully.

A basic question regarding the $\mathrm{cm}$-wave emission is whether it comes from the stellar photosphere or a somewhat larger radio photosphere. The radio emission from Mira variables comes from a radio photosphere, which is controlled by $\mathrm{H}^{-}$free-free opacity and is approximately twice the size of the stellar photosphere (defined in line-free regions of the IR spectrum) (Reid \& Menten 1997a). If a size (or stricter upper limit) could be measured with the VLA, that would directly yield a brightness temperature. A temperature below $2000 \mathrm{~K}$ would be expected for a radio photosphere, whereas a temperature in excess of 2000 would be expected for the stellar photosphere.

The amplitude of the radio variations of IRC+10216 of $\pm 25 \%$ is larger than for Mira variables, where variations of $< \pm 15 \%$ are observed by Reid \& Menten (1997a). This is in contrast to variations of $\pm 40 \%$ (see appendix) that would be expected from the stellar photosphere of IRC +10216 , based on IR variations of nearly 2 mag. For Miras, simple models of radio photospheres with shocks propagating outward with speeds less than $\approx 7 \mathrm{~km} \mathrm{~s}^{-1}$ can explain the absence of strong radio variability. However, shocks propagating outward with speeds of $\approx 10-15 \mathrm{~km} \mathrm{~s}^{-1}$ can produce variation of the magnitude we find in IRC+10216 (Reid \& Menten 1997b).

\section{Conclusions and outlook}

Using the VLA, observations of IRC+10216 at 8.4, 14.9, and $22.5 \mathrm{GHz}$ were made over a three year period. We find probable variability and a spectral index of about 1.9, i.e. near that of optically thick blackbody emission, but consistent with a radio photosphere as observed in Mira variables.
Future VLA A-configurations observations at Q-band (7 mm wavelength) with a resolution of 35 mas should definitely resolve the emission and yield a measurement of the brightness temperature. This could discriminate between a stellar and radio photosphere. Such observations may even lead to the detection of surface structure, as observed for $\alpha$ Orionis by Lim et al. (1998). These observations will benefit greatly by employing the "fast-switching" technique.

Acknowledgements. We would like to thank the referee for comments and, in particular, Malcolm Walmsley for taking the editor's job very seriously, which led to a significant improvement of the paper. This research has made use of the SIMBAD database, operated at CDS, Strasbourg, France.

\section{Appendix: Modeling IRC+10216's spectral energy distribution}

The radiative transfer in the dusty circumstellar envelope around IRC +10216 has been computed by various authors (for instance, Le Bertre 1987; Groenewegen 1997), but the most ambitious and complete modeling was performed by Men'shchikov et al. (2001) who $a$ ) considered a two-dimensional configuration to explain the non-radial structure close to the star (discovered at $2.2 \mu \mathrm{m}$ wavelength speckle interferometry by Osterbart et al. 2000), $b$ ) took into account details in the composition of the dust by adding, to the major component of amorphous carbon, admixtures of silicon carbide and magnesium sulfide grains to reproduce broad spectral features seen by the Infrared Space Satellite (ISO) and from the ground (at 11 and $~ 30 \mu \mathrm{m}$ ) and, $c$ ), included the phase in the stellar cycle. The bolometric luminosity of the star oscillates in their model by a factor of 2.5, although the average (infrared) $K$-band variations of $\sim 2$ mag (Dyck et al. 1991) would suggest a greater ratio of maximum to minimum luminosity, $L_{\max } / L_{\min } \simeq 6$.

We add to the existing radiative transfer calculations another one, on a much more modest level, which has the goal of clarifying the relative contributions of the star and its dusty envelope to the long wavelength emission. In light of the huge amount of observational data, simplicity has, despite its obvious shortcomings, the advantage of transparency and of not being entangled by occasionally secondary details. The numerical code is based on a standard ray tracing method assuming radial symmetry. It can handle an arbitrary number of grain types, includes scattering and is described in Sect. 13.2 of Krügel (2003).

Our model parameters are as follows: The star has at maximum a luminosity $L=1.1 \times 10^{4} L_{\odot}$ and emits as a blackbody of temperature $T_{*}=2000 \mathrm{~K}$. The density distribution of the dust is radially symmetric with $\rho(r) \propto r^{-2}$. The outer radius of the circumstellar envelope equals $3 \times 10^{17} \mathrm{~cm}$, corresponding to a source diameter of $6^{\prime}$ at a distance of $110 \mathrm{pc}$, the inner radius is $3 \times 10^{14} \mathrm{~cm}$, or about five stellar radii. There the dust temperature is around $1000 \mathrm{~K}$, which is close to the expected condensation temperature. The optical depth in the $V$-band toward the star amounts to $A_{\mathrm{V}}=48$ mag. Neglecting minor mineralogical components, the dust consists only of spherical amorphous carbon grains with a $n(a) \propto a^{-3.5}$ size distribution and lower and upper limits $a_{-}=80 \AA, a_{+}=640 \AA$, respectively, and optical constants as compiled by Zubko et al. (1996) for their type BE. At $1 \mathrm{~mm}$ wavelength, the mass absorption coefficient, $K_{v}$, is about $0.5 \mathrm{~cm}^{2}$ per $\mathrm{g}$ of dust with a $v^{1.6}$ frequency dependence. As the grains are much smaller than the wavelength, their size distribution does not affect the value of $K_{v}$ at FIR or longer wavelengths. Being a radiative transfer model for a dusty medium, the dust-to-gas ratio does not enter our calculations. 


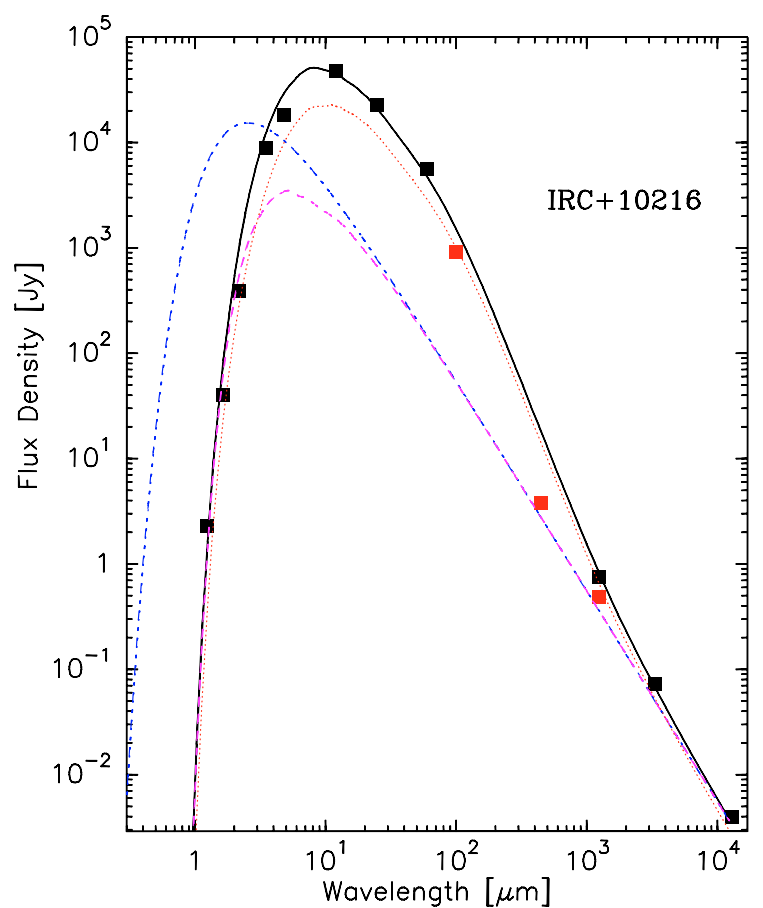

Fig. A.1. Spectral energy distribution for IRC+10216. Data (squares): $J$ - to $M$-band from Le Bertre (1987); 12 to $100 \mu \mathrm{m}$ from IRAS; $450 \mu \mathrm{m}$ from Young et al. (2004) for 2" beam; $1.24 \mathrm{~mm}$ fluxes from Lucas \& Guelin (1999), lower point for 1'.'6 beam, upper point for total envelope; $88 \%$ of the total $3 \mathrm{~mm}$ flux (same authors) come from the inner 2'. $4 ; 1.3 \mathrm{~cm}$ point, this paper. Models: the solid curve is the model described in the Appendix for maximum luminosity, the dotted line is for minimum light, when the stellar luminosity is 2.5 times weaker and the surface temperature is $1700 \mathrm{~K}$. The dash-dots, which overshoot the near IR data points, are flux densities from the star alone. The dashed line gives only the stellar flux, after extinction by the envelope of $A_{\mathrm{V}}=48 \mathrm{mag}$.

Figure A.1 displays model results together with observational data appropriate near maximum luminosity. The solid curve is our fit near maximum luminosity within a $6^{\prime}$ beam. It was obtained by adjusting the total optical depth, the inner radius, and the grain sizes, the other above mentioned parameters were kept fixed. Overall, the fit is satisfactory. The squares which lie significantly below the model refer to smaller angular sizes (see the caption of Fig. A.1).

Figure A.2 shows how the flux increases with beam size. As the curves are flat in the very left part of the figure, the emission from diameters less than 0.2 is due to the star, not to dust, despite the central dust density peak. One can read from the figure that the fraction of the total flux contributed by the stellar photosphere increases from $\sim 4 \%$ at $100 \mu \mathrm{m}$ to $\sim 96 \%$ at $1.3 \mathrm{~cm}$.

One can qualitatively understand why the brightness variations over a pulsational period are considerably smaller in the radio regime (Fig. 1) than at near infrared wavelengths. The monochromatic flux density which we would receive without foreground extinction, $F_{v}$, from a star with a blackbody photosphere of temperature $T$ and luminosity $L$ is

$F_{v}=\frac{L B_{v}(T)}{4 \sigma D^{2} T^{4}}$

where $\sigma$ denotes the Stefan-Boltzmann constant and $D$ the distance. The equation becomes obvious by integrating over frequency which yields $4 \pi D^{2} F=L$, with $F=\int F_{\nu} \mathrm{d} v$. In the

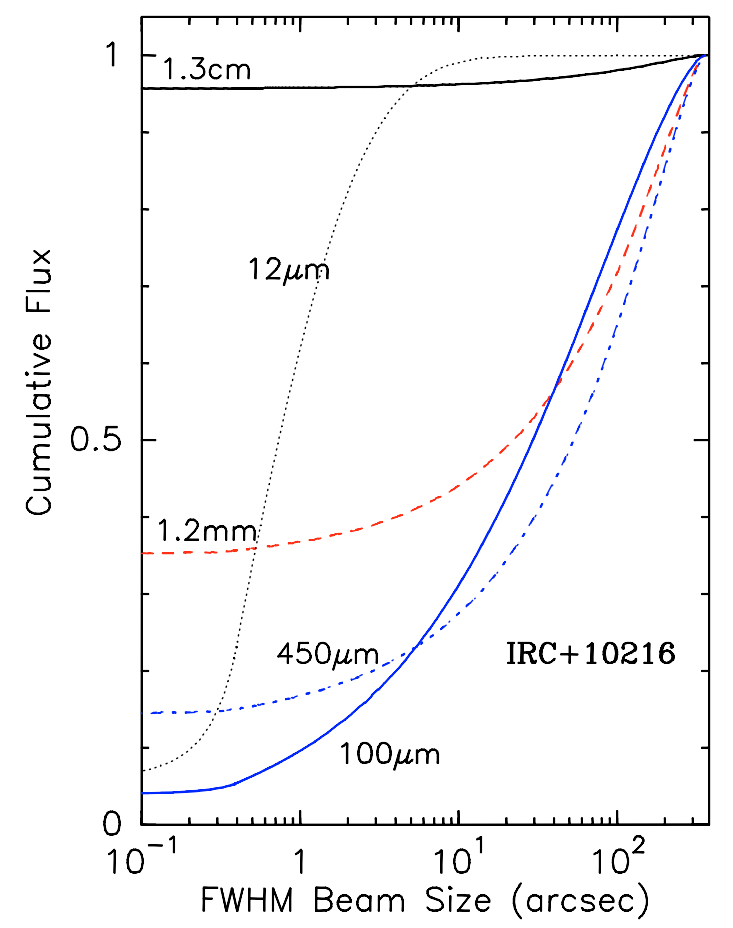

Fig. A.2. Normalized model flux density as a function of aperture at five wavelengths. The star has an assumed angular diameter of $0{ }^{\prime} 075$; at a comparable resolution essentially all the emission comes from the star. Substantial dust emission is indicated for beam sizes $>1^{\prime \prime}$ at IR wavelengths.

Rayleigh-Jeans limit, at radio wavelengths, $B_{v}(T) \propto T$ and therefore $F_{v} \propto L / T^{3}$.

How do $L$ and $T$ vary during a cycle? Reid \& Menten (1997a) in their Eq. (1), which is based on observations by Pettit $\&$ Nicholson (1933), approximate the stellar radius and temperature of a Mira variable as a function of phase $\phi$ by $T(\phi)=$ $2300+300 \cos \phi \mathrm{K}$ and $R(\phi)=2+0.4 \sin \phi$ AU. Oscillations in luminosity, $L(\phi)$, follow because $L=4 \pi R^{2} \sigma T^{4}$. According to these formulae, temperature and luminosity are roughly in phase, and therefore the observed radio flux $F_{y} \propto L / T^{3}$ changes less than $L$ itself. The radio $F_{v}$ varies from maximum to minimum by a factor of 2 (corresponding to excursions from the mean by $\pm 40 \%$ ) and $L$ varies by a factor 3.76 . We note that at infrared wavelengths, assuming a constant optical depth over the cycle and no dust emission, the monochromatic flux mimics $L$ closely. The dotted curve in Fig. A.1 is our model for IRC+10216 at minimum (2.5 times less luminous, $T=1700 \mathrm{~K}$ ). At minimum, the $1.3 \mathrm{~cm}$ radio brightness is $25 \%$ lower and the $J$ - and $H$-band flux densities are 2.5 times lower compared to maximum light.

The radio variations around the mean suggested by Fig. 1 are smaller than $\pm 40 \%$. This suggests that the cm-wave emission may not come directly from the stellar photosphere. However, other possible reasons for this discrepancy might be a smaller variability in the bolometric luminosity (by a factor 2.5 , as Men'shchikov et al. 2001 assume, and not by 3.76) or inaccuracies in the simple expressions for $R(\phi)$ and $T(\phi)$.

\section{References}

Baars, J. W. M., Genzel, R., Pauliny-Toth, I. I. K., \& Witzel, A. 1977, A\&A, 61, 99

Becklin, E. E., Frogel, J. A., Hyland, A. R., Kristian, J., \& Neugebauer, G. 1969, ApJ, 158, L133 
Cernicharo, J., Guélin, M., \& Kahane, C. 2000, A\&AS, 142, 181

Crosas, M., \& Menten, K. M. 1997, ApJ, 483, 913

Drake, S. A., Linsky, J. L., Judge, P. G., \& Elitzur, M. 1991, AJ, 101, 230

Dyck, H. M., Benson, J. A., Howell, R. R., Joyce, R. R., \& Leinert, C. 1991, AJ, 102,200

Groenewegen, M. A. T. 1996, A\&A, 305, L61

Groenewegen, M. A. T. 1997, A\&A, 317, 503

Groenewegen, M. A. T., van der Veen, W. E. C. J., \& Matthews, H. E. 1998, A\&A, 338, 491

Ho, P. T. P., Moran, J. M., \& Lo, K. Y. 2004, ApJ, 616, L1

Krügel, E. 2003, The physics of interstellar dust, IoP Series in astronomy and astrophysics (Bristol, UK: The Institute of Physics)

Le Bertre, T. 1987, A\&A, 176, 107

Le Bertre, T. 1992, A\&AS, 94, 377

Lim, J., Carilli, C. L., White, S. M., Beasley, A. J., \& Marson, R. G. 1998, Nature, 392, 575

Loup, C., Forveille, T., Omont, A., \& Paul, J. F. 1993, A\&AS, 99, 291
Lucas, R., \& Guélin, M. 1999, in Asymptotic Giant Branch Stars, IAU Symp., 191,305

Men'shchikov, A. B., Balega, Y., Blöcker, T., Osterbart, R., \& Weigelt, G. 2001, A\&A, 368, 497

Osterbart, R., Balega, Y. Y., Blöcker, T., Men'shchikov, A. B., \& Weigelt, G. 2000, A\&A, 357, 169

Perryman, M. A. C., Lindegren, L., Kovalevsky, J., et al. 1997, A\&A, 323, L49

Pettit, E., \& Nicholson, S. B. 1933, ApJ, 78, 320

Reid, M. J., \& Menten, K. M. 1997a, ApJ, 476, 327

Reid, M. J., \& Menten, K. M. 1997b, Ap\&SS, 251, 41

Richichi, A., Chandrasekhar, T., \& Leinert, C. 2003, New Astron., 8, 507

Sahai, R., Claussen, M. J., \& Masson, C. R. 1989, A\&A, 220, 92

Tuthill, P. G., Monnier, J. D., Danchi, W. C., \& Lopez, B. 2000, ApJ, 543, 284

Walmsley, C. M., Chini, R., Kreysa, E., et al. 1991, A\&A, 248, 555

Young, K. H., Hunter, T. R., Wilner, D. J., et al. 2004, ApJ, 616, L51

Zubko, V. G., Mennella, V., Colangeli, L., \& Bussoletti, E. 1996, MNRAS, 282, 1321 